Structural study of disordered $\mathrm{SiC}$ nanowires by three-dimensional rotation electron diffraction

This content has been downloaded from IOPscience. Please scroll down to see the full text.

2014 Mater. Res. Express 1045023

(http://iopscience.iop.org/2053-1591/1/4/045023)

View the table of contents for this issue, or go to the journal homepage for more

Download details:

This content was downloaded by: zhijianshen

IP Address: 77.238.49.32

This content was downloaded on 01/12/2014 at 13:11

Please note that terms and conditions apply. 


\title{
Structural study of disordered SiC nanowires by three-dimensional rotation electron diffraction
}

\author{
Duan $\mathrm{Li}^{1,2}$, Peng Guo ${ }^{1,2}$, Elisângela Guzi de Moraes ${ }^{3}$, Wei Wan ${ }^{1,2}$, Ji Zou ${ }^{1,2}$, \\ Paolo Colombo ${ }^{3,4}$ and Zhijian Shen ${ }^{1,2}$ \\ ${ }^{1}$ Department of Materials and Environmental Chemistry, Arrhenius Laboratory, Stockholm \\ University, S-106 91 Stockholm, Sweden \\ ${ }^{2}$ Berzelii Center EXSELENT on Porous Materials, Stockholm University, S-106 91 Stockholm, \\ Sweden \\ ${ }^{3}$ Dipartimento di Ingegneria Industriale, University of Padova, Via Marzolo 9, 35131 Padova, \\ Italy \\ ${ }^{4}$ Department of Materials Science and Engineering, Pennsylvania State University, University \\ Park, PA 16801, USA \\ E-mail: zhijian.shen@mmk.su.se
}

Received 11 September 2014

Accepted for publication 9 October 2014

Published 10 November 2014

Materials Research Express 1 (2014) 045023

doi:10.1088/2053-1591/1/4/045023

\begin{abstract}
The structure of disordered $\mathrm{SiC}$ nanowires was studied by using the threedimensional rotation electron diffraction (RED) technique. The streaks shown in the RED images indicated the stacking faults of the nanowire. High-resolution transmission electron microscopy imaging was employed to support the results from the RED data. It suggested that a $2 \mathrm{H}$ polytype is most possible for the nanowires.
\end{abstract}

Keywords: rotation electron diffraction, nanowire, polytype

\section{Introduction}

The last decade witnessed extensive studies on SiC based one-dimensional (1D) nanostructures due to the remarkable properties of the SiC group: high breakdown electric field, low coefficient of thermal expansion, high oxidation and corrosion resistance, high specific strength at both room temperature and elevated temperatures, as well as high thermal conductivity [1-4]. In particular, SiC nanowires (NWs) are expected to benefit unique application possibilities for nano-optical/electronic devices in harsh environments [1, 4-12]. Various synthesis routes have been explored and the growth of NWs governed by the catalyst-assisted vapor-liquid-solid (VLS) mechanism is predominant [1, 2, 6, 11-14].

It is well known that crystalline $\mathrm{SiC}$ forms up to several hundreds of different polytypes. Among them only five are frequent; three hexagonal $(2 \mathrm{H}, 4 \mathrm{H}$ and $6 \mathrm{H})$, one rhombohedral $(15 \mathrm{R})$ 
Table 1. Crystallography data for some common polytypes of SiC from ICSD database.

\begin{tabular}{|c|c|c|c|c|c|c|c|c|c|c|}
\hline \multirow[b]{2}{*}{ Polytypes } & & \multicolumn{4}{|c|}{ Unit cell parameters } & \multirow[b]{2}{*}{ Space group } & \multirow[b]{2}{*}{ Crystal system } & \multirow{2}{*}{$\begin{array}{l}d \text {-spacing }(\AA) \text { of } \\
\text { some visible reflec- } \\
\text { tions for }(h k l) \text { plane }\end{array}$} & \multirow[b]{2}{*}{$\begin{array}{l}\text { ICSD } \\
\text { No. }\end{array}$} & \multirow[b]{2}{*}{ Stacking sequence } \\
\hline & & $a$ & $b$ & $c$ & $Z$ & & & & & \\
\hline \multirow[t]{6}{*}{$\alpha-\mathrm{SiC}$} & $2 \mathrm{H}$ & 3.076 & 3.076 & 5.048 & 2 & $\begin{array}{l}P 6_{3} m c \\
\quad(\text { No.186) }\end{array}$ & Hexagonal & $\begin{array}{c}\left(\begin{array}{lll}0 & 1 & 0\end{array}\right): 2.66 \\
2.52\end{array}$ & 41487 & $\mathrm{AB}$ \\
\hline & $4 \mathrm{H}$ & 3.073 & 3.073 & 10.053 & 4 & & & $\begin{array}{c}\left(\begin{array}{llll}0 & 1 & 0\end{array}\right): 2.66\left(\begin{array}{lll}0 & 0 & 4\end{array}\right): \\
2.51 \\
\left.\begin{array}{lllll}0 & 1 & 3\end{array}\right): 2.08\end{array}$ & 24170 & $\mathrm{ABAC}$ \\
\hline & $6 \mathrm{H}$ & 3.073 & 3.073 & 15.079 & 6 & & & $\begin{array}{c}\left(\begin{array}{lll}0 & 0 & 2\end{array}\right): 7.53\left(\begin{array}{lll}0 & 0 & 6\end{array}\right): \\
2.51\left(\begin{array}{lll}0 & 1 & 5\end{array}\right): 1.99\end{array}$ & 24169 & $\mathrm{ABCACB}$ \\
\hline & $8 \mathrm{H}$ & 3.079 & 3.079 & 20.146 & 8 & & & $\begin{array}{c}\left(\begin{array}{lll}0 & 1 & 0\end{array}\right): 2.66\left(\begin{array}{lll}0 & 0 & 8\end{array}\right): \\
2.51 \\
\left(\begin{array}{llll}0 & 1 & 7\end{array}\right): 1.95\end{array}$ & 24630 & ABACABCB \\
\hline & $16 \mathrm{H}$ & 3.073 & 3.073 & 40.208 & 16 & & & $\begin{array}{r}\left(\begin{array}{lllll}0 & 1 & 0\end{array}\right): 2.66 \\
2.51\end{array}$ & 42858 & АВСВАСАВАСВСАВАC \\
\hline & $15 \mathrm{R}$ & 3.073 & 3.073 & 37.7 & 15 & $\begin{array}{l}R 3 m \\
\quad(\text { No.160) }\end{array}$ & Rhombohedral & $\begin{array}{l}\left(\begin{array}{lll}0 & 0 & 3\end{array}\right): 12.56\left(\begin{array}{ll}0 & 0 \\
15\end{array}\right): 2.51\left(\begin{array}{ll}0 & 1 \\
14) & 1.89\end{array}\right. \\
\end{array}$ & 24168 & АВСВАСАВАСВСАСВ \\
\hline$\beta-\mathrm{SiC}$ & $3 \mathrm{C}$ & 4.358 & 4.358 & 4.358 & 4 & $\begin{array}{l}F-43 m \\
\quad(\text { No.216) }\end{array}$ & Cubic & $\begin{array}{c}\left(\begin{array}{lll}1 & 1 & 1\end{array}\right): 2.51\left(\begin{array}{lll}0 & 0 & 2\end{array}\right): \\
2.17\left(\begin{array}{lll}0 & 2 & 2\end{array}\right): 1.54\end{array}$ & 28895 & $\mathrm{ABC}$ \\
\hline
\end{tabular}


and one cubic (3C) polytype structures [1, 9, 12, 13]. They differ in unit cell parameters (especially the c value), space group and stacking sequences, see table 1. Generally, SiC NWs of a specific polytype grow along a preferred direction (for $2 \mathrm{H}, 4 \mathrm{H}$ and $6 \mathrm{H}$ this is along [0001] while for $3 \mathrm{C}$ the direction is [111]) $[9,15]$.

The commonly used techniques for structural determination include powder $\mathrm{x}$-ray diffraction (PXRD), Raman spectroscopy and traditional electron crystallography. PXRD is one of the most used methods for phase composition analysis. It faces, however, difficulties when dealing with crystals of small sizes and structurally similar polytypes with frequent peak overlapping. The presence of additional phases might increase peak disturbance, for instance, in the case of $\mathrm{SiC}$ and $\mathrm{Si}_{3} \mathrm{~N}_{4}$ mixtures. Raman spectroscopy is an efficient method to determine chemical bonds of inorganic materials. Two modes, the longitudinal optical (LO) and transverse optical (TO), can be evaluated to distinguish $\mathrm{SiC}$ variants $[8,9,13,16]$. However, structure defects such as stacking faults (SFs) and micro-twins, which are often involved during the growth of SiC NWs, always result in Raman peak shifting and broadening [4, 8, 13]. In addition, the random stacking sequences of atomic planes could create a local mixture of polytypes, further leading to the observation of additional peaks in the Raman spectrum [12]. Traditional 2D electron crystallography is effective and has been widely used to identify the polytypes, defects and growth direction of $\mathrm{SiC}[17,18]$. Nevertheless, to obtain sufficient data for identification by conducting a large number of transmission electron microscopy (TEM) measurements, as well as data analysis later on, is time consuming and requires great expertise in TEM. The commonly existing SFs often result in streaked diffraction spots, further interfering with the study of the structure [12].

The recently developed three-dimensional rotation electron diffraction (3D RED) technique has shown superior advantages for both phase identification and structure determination of unknown crystals of micro- and even nano-size [19-23]. The basic principle of RED is to collect 3D electron diffraction data by combining electron beam tilt and goniometer tilt in TEM $[19,21]$. As data acquisition and further processing are automatically controlled by the RED software package, much more information can be rapidly obtained within hours down to minutes, compared with hours or days spent by conventional electron diffraction data in two dimensions. In this work, we demonstrate our attempt to study the structure of highly disordered $\mathrm{SiC}$ NWs by the RED method.

\section{Experimental procedure}

$\mathrm{Si}_{3} \mathrm{~N}_{4}$ powder (grain size $d_{50}=0.6 \mu \mathrm{m}$, purity $\geqslant 96 \mathrm{wt} \%$, containing $\mathrm{Fe}$ as catalyst, $\alpha$ phase $\geqslant 91.5 \%$ ) with the sintering additives of $5 \mathrm{wt} \% \mathrm{Y}_{2} \mathrm{O}_{3}$ (grain size $d_{50}=50 \mathrm{~nm}$, purity $\geqslant 99.95 \mathrm{wt} \%$ ) and $5 \mathrm{wt} \% \mathrm{MgO}$ (particle size $d_{50}=4.6 \mu \mathrm{m}$, purity $\geqslant 99.99 \mathrm{wt} \%$ ) were used for fabricating the substrate. To prepare the porous substrate, a surfactant-based foaming process was employed. The starting powders were mixed and ball milled in ethanol for $4 \mathrm{~h}$. After drying and sieving, the powder mixture was added stepwise to deionized water containing $1 \mathrm{wt} \%$ (relative to the powder mixture) dispersing agent polyacrylic acid (PAA, Sigma-Aldrich Sweden AB, Stockholm, Sweden) under constant stirring. Subsequently, the $\mathrm{Si}_{3} \mathrm{~N}_{4}$ slurry was homogenized and dispersed by ball milling for $2 \mathrm{~h}$. The introduction of gas bubbles took place by adding 50 vol\% octane $\left(\mathrm{C}_{8} \mathrm{H}_{18}\right.$, Sigma-Aldrich Sweden $\mathrm{AB}$, Stockholm, Sweden $)$ and $0.22 \mathrm{vol} \%$ (with regard to the suspension) nonionic surfactant polysorbate Tween ${ }^{\circledR} 80$ (VWR 
International Ltd, Bedfordshire, UK) with stirring at $700 \mathrm{rpm}$ for $3 \mathrm{~min}$. Afterwards, pore evolution was aided by octane droplet evaporation at ambient air for $24 \mathrm{~h}$. The evaporation of the oil (alkane) phase and concurrent drying (water evaporation) enabled the transition of the emulsified suspensions into green solid $\mathrm{Si}_{3} \mathrm{~N}_{4}$ substrate. The $2 \mathrm{H} \mathrm{SiC}$ nanowires were grown on the porous $\mathrm{Si}_{3} \mathrm{~N}_{4}$ substrate by heat treatment in a modified spark plasma sintering (SPS) set-up (Dr Sinter 2050, Sumitomo Coal Mining Co., Tokyo, Japan) under vacuum. The green substrate was loaded in a covered cylindrical graphite crucible with an inner diameter of $50 \mathrm{~mm}$ and outer diameter of $70 \mathrm{~mm}$, protected by a $\mathrm{Si}_{3} \mathrm{~N}_{4}$ powder bed and isolated by graphite felts. The temperature was automatically raised to $600^{\circ} \mathrm{C}$ over a period of $5 \mathrm{~min}$, and from there onwards it was monitored and regulated by an optical pyrometer focused on the wall centrally inside the crucible through a hole of $\sim 5 \mathrm{~mm}$ in diameter. The substrate was heated to $1500{ }^{\circ} \mathrm{C}$ with a heating rate of $50{ }^{\circ} \mathrm{C} \mathrm{min}^{-1}$ and maintained for $10 \mathrm{~min}$. Then the temperature was increased $1600^{\circ} \mathrm{C}$ at $50^{\circ} \mathrm{C} \mathrm{min}^{-1}$ and was finally held for $3 \mathrm{~min}$.

For structural study of the NWs, the sample was crushed into powder and dispersed in absolute ethanol with ultrasonification for $5 \mathrm{~min}$. A droplet of the suspension was transferred onto a copper grid. HRTEM/SAED/EDX and RED were carried out on a Schottky-type field emission transmission electron microscope (TEM, JEM-2100F, JEOL Ltd, Tokyo, Japan) and a $\mathrm{LaB}_{6}$ based transmission electron microscope (TEM, JEM-2100, JEOL Ltd, Tokyo, Japan) operated at $200 \mathrm{kV}$ using a double-tilt tomography sample holder, respectively. The 3D RED data were collected from one selected $\mathrm{SiC} \mathrm{NW}$ at the tilt range of $-32 \sim+41^{\circ}$ with a tilt step of $0.5^{\circ} .1 \mathrm{~s}$ per ED pattern was used for exposure and $>180$ SAED patterns could be collected in $\sim 30$ min. Both data aquisition and processing were automatically controlled by the RED software package.

\section{Results and discussion}

The geometrical structure and the energy-dispersive x-ray spectroscopy (EDX) compositions within the examined NWs specimen are illustrated in figure 1 . As seen, the diameter of the NW is $\sim 200 \mathrm{~nm}$ and it possesses a tip-body structure with the presence of a semispherical cap at the top. The EDX analysis result inserted suggests that the chemical composition of the tip is $\mathrm{SiFe}_{1.22}$ and that the fiber body is pure $\mathrm{SiC}$. The $\mathrm{Cu}$ signal in the EDX spectra came from a supporting grid. These observations confirm that the growth of $\mathrm{SiC} \mathrm{NWs} \mathrm{follows} \mathrm{the} \mathrm{vapor-}$ liquid-solid (VLS) mechanism with the aid of Fe catalysis [1, 2, 6, 11-13].

In order to study the disordered structure of the SiC NWs, the RED technique was employed [21]. The general procedure is described below (see figure 2): after a routine alignment of the microscope and adjusting the eucentric height of the crystal, RED data was collected using the RED data collection software. Two tilt modes, namely beam tilt (BT) and goniometer tilt (GT), were combined together in the data collection [21]. BTs were recorded with a fine angle step length $\left(0.2^{\circ}\right)$ while GTs used a coarser step length $\left(2^{\circ}\right)$ along a common tilt axis. Thus, the final RED data contained $>180$ frames and covered a tilt range from $-32^{\circ}$ to $+41^{\circ}$. The subsequent data processing was done by the RED data processing software, including shift correction of the ED frames, peak identification from observed diffraction spots in individual ED frames and reconstruction of the 3D reciprocal space. The reconstructed reciprocal space was examined using the visualizer included in the software. 


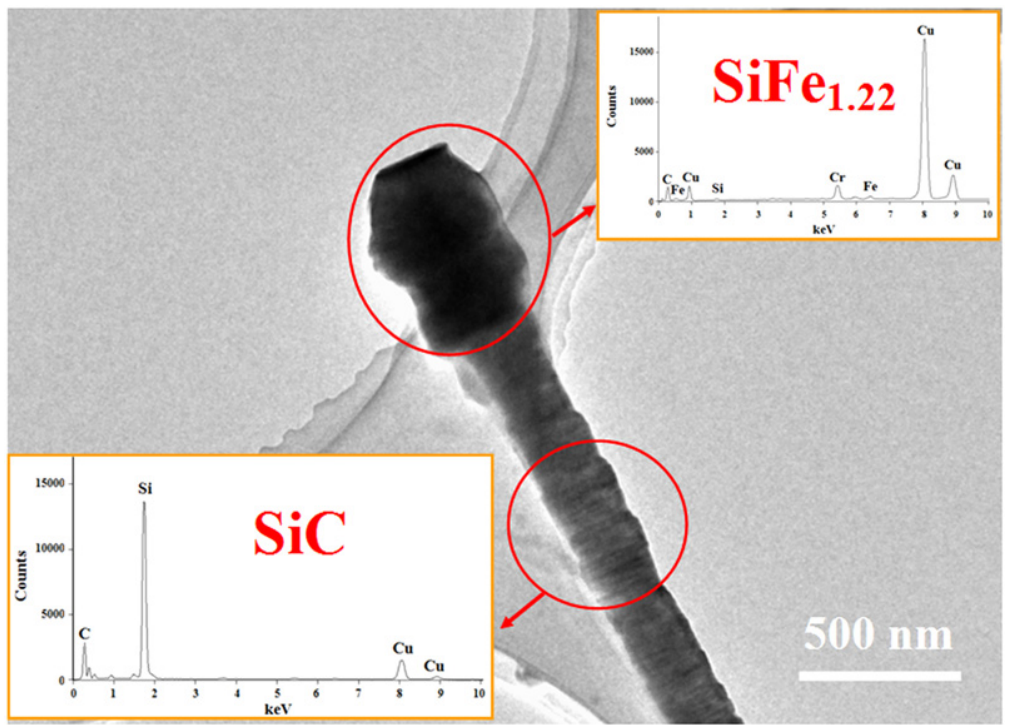

Figure 1. TEM image of one selected SiC NW. The NW has a tip-body structure. The insets are EDX spectra, revealing that the composition of the tip and body is $\mathrm{SiFe}_{1.22}$ and $\mathrm{SiC}$, respectively.

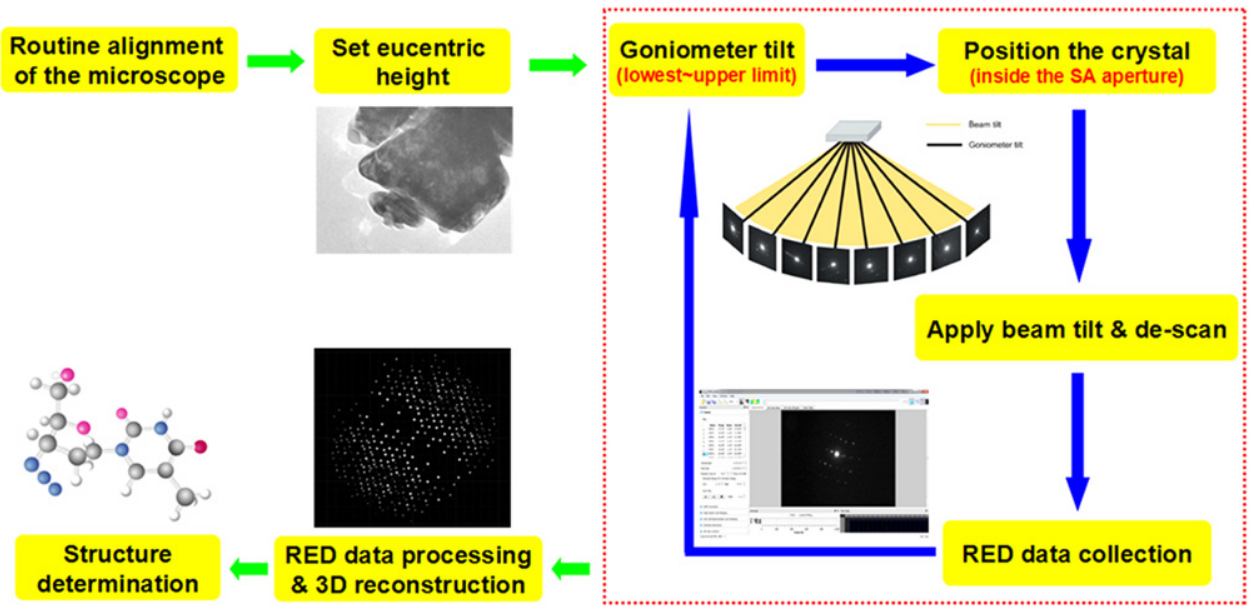

Figure 2. Schematic representation of the concept of the RED method and the procedure for structure determination.

The RED results are summarized in figures 3(a)-(c). The TEM area containing the selected NW for data collection is shown as an inset in figure 3(a). The 2D projections of the reconstructed 3D ED patterns along two directions which are perpendicular to each other are shown in figures 2(a) and (b), respectively. It can be seen from figure 3(a) that there are diffuse lines in the $3 \mathrm{D}$ electron diffraction data, indicating stacking disorder. A hexagonal lattice is observed in figure 3(b). The length value of $a^{*}$ and $b^{*}$ (in reciprocal space) were measured to be $0.38 \AA^{-1}$, and the angle between them is $\sim 60^{\circ}$. These facts are consistent with the electron diffraction pattern from the $\mathrm{Si}-\mathrm{C}$ layer that builds up all the different polytypes of $\mathrm{SiC}$. The diffuse lines seen in figure 3(a) can be explained by the stacking disorder of this Si-C layer. In 


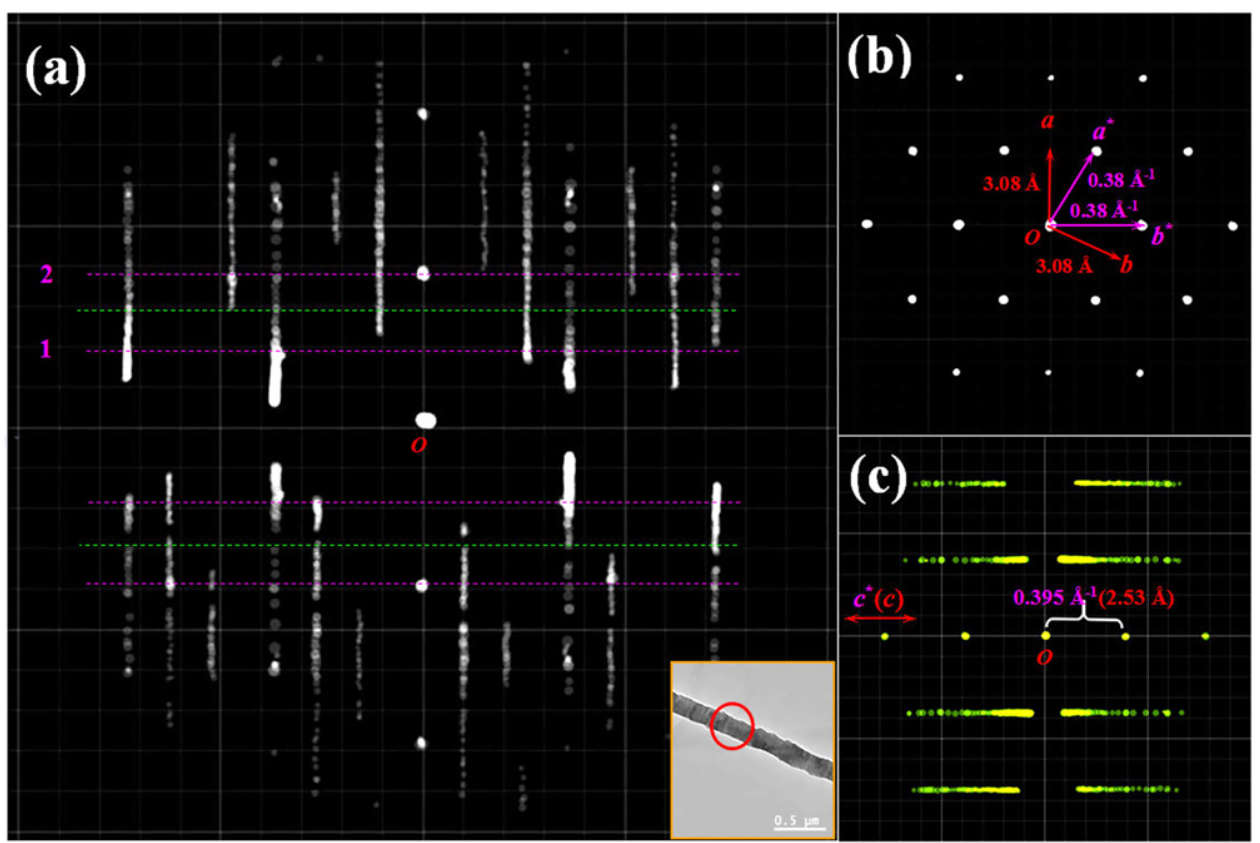

Figure 3. Results from RED data collected from the selected SiC NW: (a) a 2D projection of the reconstructed 3D ED pattern, showing diffuse lines and a central line of sharp reflection spots. The pink dash lines represent the areas for diffraction spots of a $2 \mathrm{H} \mathrm{SiC}$ polytype, while the green lines indicate the diffraction spots of a $4 \mathrm{H}$ (or $8 \mathrm{H}$, $16 \mathrm{H} . .$.$) SiC polytype. The inset is a TEM image of the selected NW for data collection$ where the area marked with red circle was measured. (b) a $2 \mathrm{D}$ projection of the reconstructed 3D ED pattern along the diffuse lines. (c) a 2D slice cut from the reconstructed 3D reciprocal lattice, showing the sharp reflection spots on the central line. The smallest scale of the white lattice is $0.1 \AA^{-1}$.

order to identify the polytype of the $\mathrm{SiC} \mathrm{NW}$, we need to determine the periodicity of the $\mathrm{Si}-\mathrm{C}$ stacking. Figure 3(c) shows the 2D slice cut from the reconstructed 3D reciprocal lattice. We can see that the central line of reflections contains sharp reflections while all the other lines are diffuse. The sharp reflections correspond to lattice planes with a $d$-spacing of $\sim 2.53 \AA$, which matches the $d$-spacings of the $\mathrm{Si}-\mathrm{C}$ layers in all $\mathrm{SiC}$ polytypes. Reflections corresponding to a layer distance of multiples of $2.53 \AA$ were observed, revealing that the polytype of this NW is either $3 \mathrm{C}$ or $2^{n} \mathrm{H}$ as otherwise we would have observed reflections with $d$-spacings of multiples of $2.53 \AA$.

In order to distinguish the most probable polytypes among $3 \mathrm{C}$ and the $2^{n} \mathrm{H}$ group, we need to examine reflections other than those located on the central line from figure 3(a). Polytypes of $3 \mathrm{C}$ and $2^{n} \mathrm{H}$ are built by stacking the Si-C layer with a periodicity of 3 or $2^{n}$. As a result, the first reflection (closest to the 000 reflection) on the central line will be 003 or $002^{n}$, and there will be reflections with indices $h k 1, h k 2, h k 3 \ldots$, up to $h k 2^{n}$, which form layers perpendicular to the stacking direction. In the present case, although the serious stacking disorder made the reflection diffuse, it was observable that the diffuse intensities are higher at certain positions on the diffuse lines, as indicated by the pink dash lines in figure 3(a). These correspond to the $h k 1$ (line 1) and $h k 2$ (line 2) reflections of polytype $2 \mathrm{H}$. The green lines in figure 3 (a) mark the positions that would correspond to the $h k 3$ reflections of the $4 \mathrm{H}$ polytype. The absence of higher 


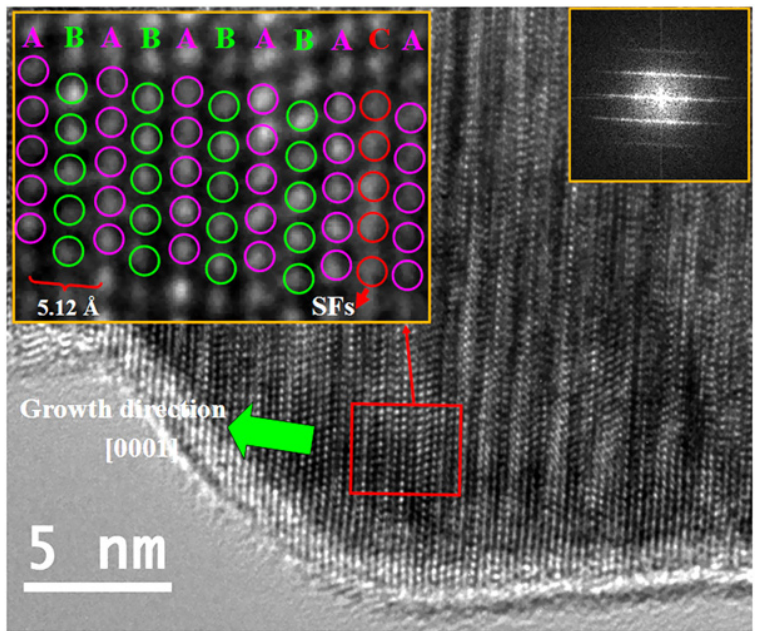

Figure 4. HRTEM image of a SiC NW showing the stacking sequence. The top-right inset is its FFT pattern. The diffuse reflections indicate a disordered structure. The topleft inset is the enlarged HRTEM image with markings of the stacking of the $2 \mathrm{H}$ polytype with SFs. The growth direction of the NW is along [0001] axis.

diffuse intensities at this line, and also at other positions, indicates that the polytype of the NW can neither be $4 \mathrm{H}$ nor others. On this account, the most possible polytype of the SiC NWs without disorder is the $2 \mathrm{H}$ member with space group $P 6_{3} m c$ (ICSD No. 41487).

For confirmation, HRTEM was performed and the image is shown in figure 4. It was taken from a small fraction of the same area used for the RED evaluation. The top-right inset displays the corresponding fast Fourier transformed (FFT) diffraction pattern. The streaks imply again disordered polytype structures of the observed NW. An enlarged HRTEM image of the SiC NW, overlaid by a two-dimensional projected atomic structural model, is displayed as the topleft inset. In the HRTEM image, the stacking order can be determined by following the stacking of the white dots. It reveals that the dominating stacking sequence is ...ABABAB ..., with the SFs distributing in a random order like ...ABABABABACA.... Here, A and B represent two basic structure modules of tetrahedral bilayers in SiC, while C suggests the SFs [24]. The distance between stacking periods is $\sim 5.12 \AA$, which is coherent with the $c$ value of $2 \mathrm{H} \mathrm{SiC}$ (see table 1). The HRTEM image taken from this small area shows only local configuration of the stacking, while RED was performed in a much larger area and provided information concerning the overall structure.

\section{Conclusion}

In summary, the 3D RED technique was employed to study the polytype of $\mathrm{SiC}$ NWs grown on a porous $\mathrm{Si}_{3} \mathrm{~N}_{4}$ substrate. The $2 \mathrm{H}$ type, with highly stacking faults, was most possible according to the obtained 3D RED data. The RED technique is promising for investigating the disordered $\mathrm{SiC}$ polytypes as it provides $3 \mathrm{D}$ data. 


\section{Acknowledgements}

Financial support of this work by European Commission through the Marie-Curie ITN project 'FUnctional Nitrides for Energy Applications, FUNEA' (FP7-PITN-GA-2010-264873) is gratefully acknowledged. The support of the Swedish Research Council (VR) \& the Swedish Governmental Agency for Innovation Systems (VINNOVA) through the Berzelii Center EXSELENT is appreciated.

\section{References}

[1] Krishnan B, Thirumalai R V K G, Koshka Y, Sundaresan S, Levin I, Davydov A V and Merrett J N 2011 Cryst. Growth Des. 11538

[2] Li Z J, Zhang J L, Meng A and Guo J Z 2006 J. Phys. Chem. B 11022382

[3] Li Z J, Li H J, Chen X L, Meng A L, Li K Z, Xu Y P and Dai L 2003 Appl. Phys. A 76637

[4] Kim H Y, Park J and Yang H 2003 Chem. Commun. 2003 256-7

[5] Li Y B, Dorozhkin P S, Bando Y and Golberg D 2005 Adv. Mater. 17545

[6] Cai Z L, Wang G, Song B, Wu R, Li J and Jian J K 2013 Cryst. Eng. Comm. 155082

[7] Li Z, Zhang M and Meng A 2011 Cryst. Eng. Comm. 134097

[8] Wu R B, Yang G Y, Gao M X, Li B S, Chen J J, Zhai R and Pan Y 2009 Cryst. Growth Des. 9100

[9] Sundaresan S G, Davydov A V, Vaudin M D, Levin I, Maslar J E, Tian Y L and Rao M V 2007 Chem. Mater. 195531

[10] Yang W, Araki H, Tang C C, Thaveethavorn S, Kohyama A, Suzuki H and Noda T 2005 Adv. Mater. 171519

[11] Yang G, Wu R, Chen J, Pan Y, Zhai R, Wu L and Lin J 2007 Nanotechnology 18155601

[12] Zekentes K and Rogdakis K 2011 J. Phys. D 44133001

[13] Yao Y, Lee S T and Li F H 2003 Chem. Phys. Lett. 381628

[14] Li B, Song Y C, Zhang C R and Yu J S 2014 Ceram. Int. 4012613

[15] Li X, Chen X and Song H 2011 Mater. Sci. Eng. B 17687

[16] Bechelany M, Brioude A, Cornu D, Ferro G and Miele P 2007 Adv. Funct. Mater. 17939

[17] Zhang Y, Han X, Zheng K, Zhang Z, Zhang X, Fu J, Ji Y, Hao Y, Guo X and LWang Z 2007 Adv. Funct. Mater. 173435

[18] Han X D, Zhang Y F, Liu X Q, Zhang Z, Hao Y J and Guo X Y 2005 J. Appl. Phys. 98124307

[19] Zhang D, Oleynikov P, Hovmöller S and Zou X 2010 Z. Kristallog 22594

[20] Martinez-Franco R, Moliner M, Yun Y, Sun J, Wan W, Zou X and Corma A 2013 Proc. Natl. Acad. Sci. 1103749

[21] Wan W, Sun J, Su J, Hovmoller S and Zou X 2013 J. Appl. Crystallogr. 461863

[22] Zhang Y B, Su J, Furukawa H, Yun Y, Gandara F, Duong A, Zou X and Yaghi O M 2013 J. Am. Chem. Soc. 13516336

[23] Willhammar T, Yun Y and Zou X 2014 Adv. Funct. Mater. 24182

[24] Daulton T L, Bernatowicz T J, Lewis R S, Messenger S, Stadermann F J and Amari S 2003 Geochim. Cosmochim. Ac. 674743 